\title{
MESOZOOPLÂNCTON DO ESTUÁRIO DO RIO DOS PASSOS, PERNAMBUCO, BRASIL
}

Recebido em: julho/2013

Aceito em: agosto/2013

\author{
Lucas Guedes Pereira FIGUEIRÊDO ${ }^{1}$ \\ Valdylene Tavares Pessoa FIDELIS ${ }^{1}$ \\ Eveline Pinheiro de AQUINO ${ }^{2}$ \\ Sigrid NEUMANN-LEITÃO ${ }^{3}$ \\ José Zanon de Oliveira PASSAVANTE ${ }^{3}$
}

\section{RESUMO}

A dinâmica estuarina atua constantemente modelando a estrutura das comunidades zooplanctônicas, portanto, foi alvo deste trabalho avaliar as características do mesozooplâncton em um estuário tropical. Para tanto, foram definidos quatro estações de coleta, as quais foram visitadas em três meses de cada período do ano (estiagem e chuvoso) para a coleta e obtenção dos dados de clorofila-a, salinidade, temperatura, profundidade e transparência, além do uso de rede de plâncton $(120 \mu \mathrm{m})$ para amostragem do mesozooplâncton. Os dados abióticos foram tratados através de ANOVA de um fator. Os

resultados abióticos encontrados caracterizam o estuário como de moderada produção, pouca profundidade e considerável influência marinha. Este último fator pôde ser observado na estrutura do mesozooplâncton, no qual ocorreram predominantemente espécies eurihalinas tipicamente estuarinas (Pseudodiaptomus e Oithona) e costeiras de altas salinidades (Favella ehrenbergii). Foi possível observar também a predominância de náuplios e estágios prematuros (copepoda), além da ocorrência de certos taxa durante os dois períodos do ano, indicando sua reprodução na área e, portanto, a saúde do ambiente.
\end{abstract}

Palavras chave: estuário tropical, dinâmica estuarina, comunidade mesozooplanctônica.

\section{ABSTRACT}

Estuarine dynamics acts continuously over the zooplankton community structure. therefore, the goal here was to assess the mesozooplankton characteristics on a tropical estuary. To do so, four stations were stated and sampled during three months at the dry season and three months at the rainy season. Water was sampled using a kitahara bottle in order to assess the salinity, temperature, chlorophyll a. Besides of that, was measured each station depth and water transparency. The mesozooplakton was sampled using a $120 \mu \mathrm{m}$ plankton net. The abiotic data were statistically analyzed applying an one way ANOVA test. The results found for the abiotic data features the estuary as of moderate production, shallow and under strong marine influence. This last factor could be observed in the mesozooplankton structure, in which occurred predominantly, eurihaline species typical of estuary (Oithona and Pseudodiaptomus) and coastal high salinity species (Favella ehrenbergii). It was also observed a predominant occurrence of nauplii and premature stage (copepodits), and the occurrence of certain taxa during both seasons of the year, indicating their reproduction on the area, and therefore the environmental health.

Key words: tropical estuary, estuary dynamics, mesozooplankton community.

\section{INTRODUÇÃo}

Os estuários são ambientes complexos cuja dinâmica é regida por fatores ambientais diversos tais como a salinidade, temperatura, sedimentação e turbidez, que estão submetidos a influência das variações periódicas da maré (TUNDISI, 1970), ao balanço entre a frente marinha e a fluvial, além das mudanças climáticas anuais que, em áreas tropicais, estão

1 - Universidade Federal de Pernambuco/ Programa de Pós-Graduação em Oceanografia/ Laboratório de Zooplâncton

2 - Universidade Federal de Pernambuco/ Programa de Pós-Graduação em Oceanografia/ Laboratório de Fitoplâncton

3 - Departamento de Oceanografia

Contatos: figueiredo@lgp.net.br; valdylenetp@yahoo.com.br; sigridnl@uol.com.br; passavante@gmail.com. 
FIGUEIRÊDO. L. G. P. et AL. Mesozooplâncton do estuário do rio dos Passos, Pernambuco, Brasil.

basicamente relacionadas a pluviometria (ESKINAZI-LEÇA et al., 1984; SASSI, 1991).

Os estuários sofrem ainda com o impacto antrópico causado pelo lançamento de contaminantes, aquicultura, turismo e pesca. Desta forma, as características de determinado estuário resultam da variedade de interrelações de fatores bióticos e abióticos, naturais ou antrópicos e seus componentes estruturais, atuantes nestes ecossistemas sob processos físicos, químicos e biológicos intensos (FLINDT et al., 1999; KIBIRIGE; PERISSINOTTO, 2003; TELESH, 2004). Como resultado desta complexidade, ocorrem flutuações na dinâmica de populações biológicas, particularmente as planctônicas, de forma que variações espaço-temporais e tipos de habitats estão entre os mais importantes fatores que afetam os padrões de abundancia de espécies, composição e estrutura do plâncton estuarino (KENNISH, 1990; HOFFMEYER, 2004).

O mesozooplâncton $(0,2-2 \mathrm{~mm})$ é um componente chave em ecossistemas costeiros, atuando como link entre a alça microbiana e a teia trófica clássica, transferindo energia e matéria dos produtores primários para níveis tróficos mais altos (CHEN et al., 2011). A composição e abundância do mesozooplâncton em águas costeiras exibem padrões temporais e espaciais devido a alta variabilidade nas propriedades hidrológicas (RAMFOS et al., 2006). Neste sentido, a temperatura e a salinidade são variáveis que influenciam diretamente no crescimento tanto do fitoplâncton como do mesozooplâncton, se tornando, portanto, fatores determinantes sobre os padrões de distribuição sazonais e espaciais do mesozooplâncton (BADYLAK; PHILIPS, 2008)

Portanto, este trabalho teve como objetivo caracterizar a comunidade mesozooplanctônica do estuário do rio dos Passos analisando a influência dos fatores ambientais e da sazonalidade sobre os padrões estruturais desta comunidade.

\section{ÁREA DE ESTUDO}

A Área de Proteção Ambiental de Guadalupe abrange aproximadamente quarenta e quatro mil hectares, nos quais fazem parte tanto áreas continentais (manguezais, mata atlântica, restingas) como marítimas (estuários, recifes) (CPRH, 1998). A esses recursos hídricos estão associados ecossistemas essenciais à manutenção, com qualidade, da vida das populações locais, dependendo da preservação dos referidos ecossistemas a sustentabilidade da área (CPRH, op cite).

Criada pelo Decreto Estadual no. 19.635, de 13 de março de 1997, a Área de Proteção Ambiental (APA) de Guadalupe localiza-se no Litoral Sul do Estado de Pernambuco, e é onde o estuário do rio dos Passos esta inserido ( $\mathrm{CPRH}$, op cite).

O rio dos Passos sofre grande influência marinha por estar inserido em um ambiente de mesomaré. Por outro lado, o mesmo está inserido no complexo estuarino para o qual convergem também o rio Formoso, Ariquindá e União, estabelecendo desta forma, outra frente que atua no estuário com o fluxo de água doce derivada da drenagem continental (Fig. 1).

O clima na região, de acordo com a classificação de Köppen se encaixa no tipo tropical quente e úmido, com temperaturas médias anuais em torno de $25,2^{\circ} \mathrm{C}$ e pluviosidade média entre 1500 a mais de $2000 \mathrm{~mm}$. 


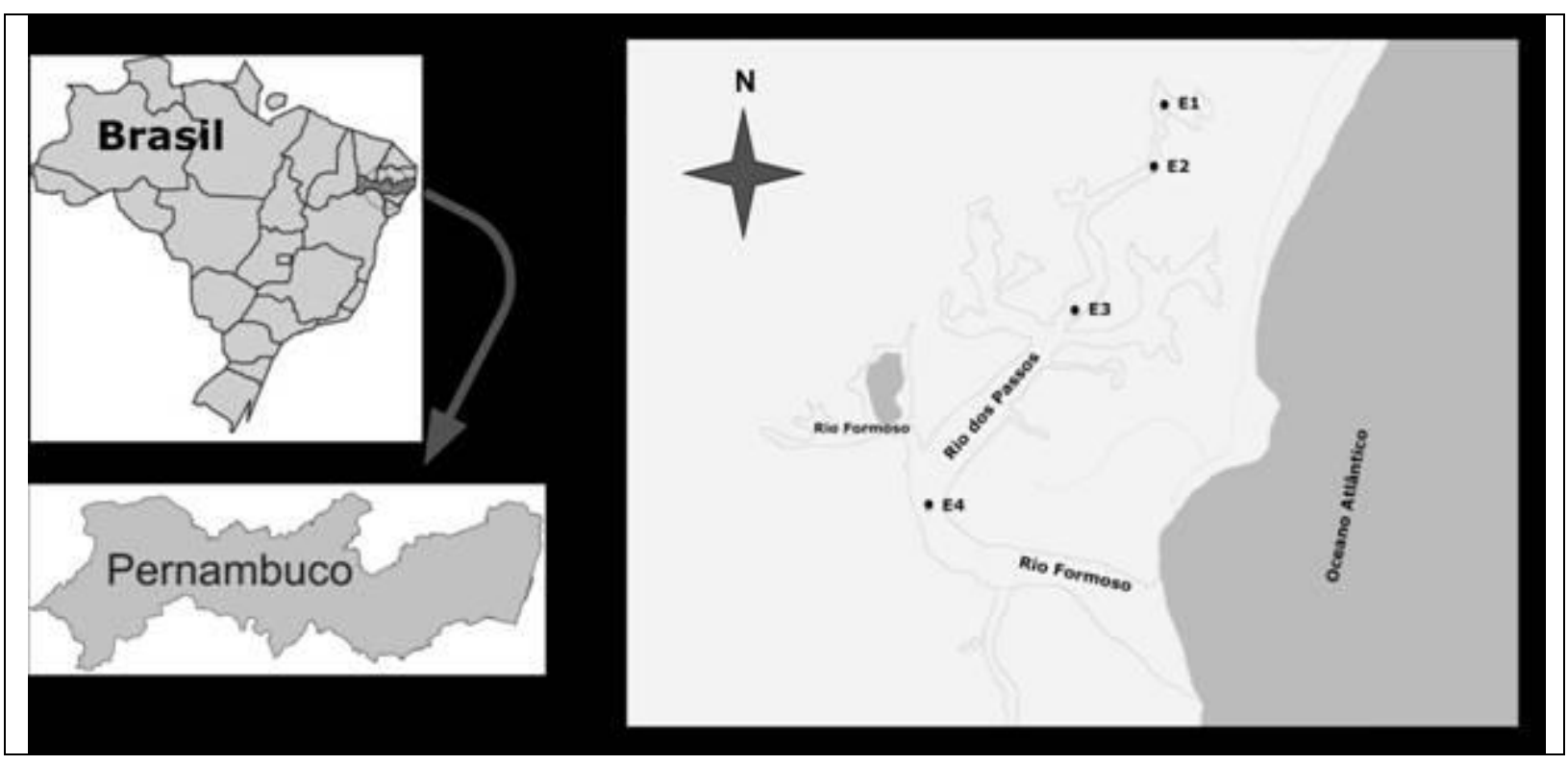

Figura 1 - Mapa do estuário do rio dos Passos, localizado no nordeste do Brasil. Estações de coleta estão indicadas em E1, E2, E3 e E4 (Fonte: Google Earth, 2012).

\section{MATERIAIS E MÉTODOS}

Foram definidos quatro estações de coletas (E1, E2, E3 e E4) com o uso de um GPS, levando-se em conta o melhor aproveitamento da altura da maré e possibilitando analisar as variações ao longo de todo o estuário. Foram realizadas coletas nos períodos seco, de dezembro de 2009 a fevereiro de 2010, e chuvoso, de junho a agosto de 2010, nas baixa-mares de sizígia.

Os dados de precipitação pluviométrica para os meses de estudo foram adquiridos do Laboratório de Meteorologia do Instituto de Tecnologia de Pernambuco (LAMEPE/ ITEP), e são referentes a estação de Cucau, localizada no próprio município de Rio Formoso.

Amostras da água recolhidas utilizando-se uma garrafa de Kitarrara foram empregadas para posterior determinação da salinidade e temperatura, as quais foram, respectivamente, aferidas com o auxilio de refratômetro e termômetro digital na ocasião da coleta.

A profundidade foi medida através de uma sonda manual, fixada a um cabo graduado. A medida da transparência da água foi obtida por meio da profundidade de desaparecimento do disco de Secchi e posteriormente foi calculado o coeficiente de extinção da luz, utilizando-se a fórmula de Poole e Atkins (1929).

Para a concentração de clorofila-a, as amostras de água acondicionadas em recipientes opacos foram filtradas logo após a coleta com auxílio de uma bomba a vácuo e colocadas em solução de cetona $90 \%$ para a extração da clorofila-a, e posterior análise espectrofotométrica, segundo Strickland e Parsons (1972), descrita pela UNESCO (1966).

Uma rede de plâncton cônica, com abertura de malha de $120 \mu \mathrm{m}, 30 \mathrm{~cm}$ de diâmetro de boca e $1 \mathrm{~m}$ de comprimento foi utilizada na coleta do zooplâncton. Os arrastos foram realizados subsuperficialmente no sentido contrário a corrente com duração de cinco minutos.

Para a análise qualitativa e quantitativa, as amostras coletadas foram examinadas em lâminas do tipo Sedwick-Rafter sob microscópio composto Zeiss, sendo a classificação zoológica dos organismos adotada a partir de literatura especializada (TREGOUBOFF; ROSE, 1957; BJÖRNBERG, 1981; BOLTOVSKOY, 1981; 1999); e HUYS e BOXSHALL (1991) assim também como a sua identificação. Para evidenciar a estrutura da comunidade e sua complexidade foram calculados os índices de Diversidade Específica (SHANNON, 1948), e grau de equitabilidade. Foram calculadas ainda, a densidade, a abundância relativa e a frequência de ocorrência. 
FIGUEIRÊDO. L. G. P. et AL. Mesozooplâncton do estuário do rio dos Passos, Pernambuco, Brasil.

\section{RESULTADOS}

Como característico do período seco, os meses de dezembro de 2009 a fevereiro de 2010 registraram uma média de chuvas de $114,8 \mathrm{~mm}$, com mínima de $95,7 \mathrm{~mm}$ no mês de fevereiro e máxima de 145,7 no mês de janeiro. Em contrapartida, no período de chuvas, a média pluviométrica atingiu 290,83 mm, com mínima de 127 no mês de agosto e máxima de 592,5 no mês de junho. A diferença entre os períodos de estiagem e chuvoso pode ser vista na tabela 1 , na qual encontram-se os principais dados de todas as variáveis.

A salinidade não apresentou diferença significativa entre as estações de coleta $(p=0,664)$. Porém, os valores revelaram um gradiente crescente da estação mais interna, onde se obteve uma média de 23,83, até a estação sob maior influência marinha, com média de 28,33 . Em relação ao período do ano, o valor máximo obtido no período chuvoso não alcançou o mínimo obtido no período seco. Desta forma, a salinidade apresentou diferença significativa, com $\mathrm{p}=<0,001$.

A temperatura, assim como a salinidade, não apresentou diferença espacial significativa $(p=0,846)$, apresentando médias próximas entre as estações, com máxima $28,15^{\circ} \mathrm{C}$ na estação 1 e mínima $27,30^{\circ} \mathrm{C}$ na estação 4 . Houve, porém, diferença sazonal significativa $(p=<0,001)$, com os maiores valores ocorrendo no período de estiagem.

A transparência apresentou diferença significativa apenas espacialmente $(p=<0,001)$, não havendo diferenças sazonais $(p=0,090)$. A estação 1 foi onde ocorreu a menor média $(0,94 \mathrm{~m})$, já na estação 4 foi registrada a maior média $(1,99 \mathrm{~m})$, determinando assim um gradiente crescente deste parâmetro em direção ao oceano.

A clorofila-a apresentou o teor máximo de $21,3 \mathrm{mg} \cdot \mathrm{m}^{-3}$ no ponto de menor influência marinha (P1), no mês de junho de 2010, durante a estação chuvosa. Já a concentração mínima $\left(1,22 \mathrm{mg} \cdot \mathrm{m}^{-3}\right)$ foi registrada no ponto sob maior influência marinha (P4), no mês de dezembro de 2009, durante a estiagem. Houve diferença significativa tanto entre as estações de coleta $(p=<0,001)$ como entre os períodos de chuvas e de estiagem $(p=0,040)$.

Tabela 1 - Valores máximos, mínimos e as médias dos parâmetros ambientais do estuário do rio dos Passos nos diferentes períodos climáticos.

\begin{tabular}{lrrr|rrr}
\hline \multicolumn{1}{c}{ VARIÁVEIS } & \multicolumn{3}{c|}{ ESTIAGEM } & \multicolumn{3}{c}{ CHUVOSO } \\
& MÁx & MÍN & MÉDIA & MÁX & MÍN & MÉDIA \\
\hline Pluviosidade $(\mathrm{mm})$ & 145,70 & 95,70 & 114,80 & 592,5 & 127,00 & 290,80 \\
Salinidade & 35,00 & 28,00 & 31,40 & 27,0 & 14,00 & 21,20 \\
Temperatura $\left({ }^{\circ} \mathrm{C}\right)$ & 31,10 & 28,00 & 29,20 & 28,0 & 24,90 & 26,10 \\
Profundidade $(\mathrm{m})$ & 4,54 & 2,20 & 3,05 & 4,77 & 2,23 & 3,18 \\
Transparência $(\mathrm{m})$ & 2,30 & 0,90 & 1,70 & 1,92 & 0,8, & 1,30 \\
Coeficiente de atenuação da luz & 1,85 & 0,72 & 1,11 & 2,21 & 0,89 & 1,33 \\
Clorofila-a $\left(\mathrm{mg}^{-3}\right.$ ) & 9,14 & 1,22 & 3,82 & 21,30 & 2,00 & 6,82 \\
\hline
\end{tabular}

\section{Mesozooplâncton}

Foram registrados 28 taxa (Tab. 2) podendo-se destacar os Copepoda, com 10 espécies, além dos indivíduos que não puderam ser identificados até o nível mais específico (Harpacticoida, Monstriloida e copepoditos). Foram registrados 25 taxa para o período seco e 22 para o período chuvoso. Na área de coleta, o maior número de taxa (22) foi registrado nas duas estações intermediárias (E2 e E3), em contrapartida dos 20 taxa registrados em E1 e E4.

Com relação a frequência de ocorrência, foram considerados muito frequentes, com mais de 60\%, Foraminifera, Favella ehrenbergii, Parvocalanus crassirostris, Oithona (copepodito), Oithona oswaldocruzi, Apocyclops sp. e Náuplios (Tab. 2). Dentre os menos frequentes estão 
Paracalanus nanus, Pseudodiaptomus richardi, Pseudodiaptomus acutus, Monstriloida, Bivalve (véliger) e Amphipoda.

Os valores de diversidade específica foram considerados baixos (Fig. 2). A maior diversidade encontrada para o período de estiagem foi de 1,59 bits.ind $^{-1}$ na estação E1, e para 0

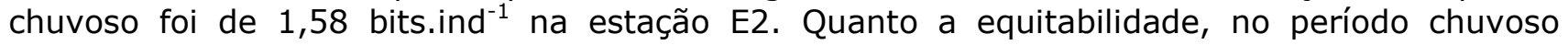
demonstrou-se uma distribuição homogênea do número de indivíduos para os taxa, com valores menores que 0,5 apenas nas estações E2 em junho e E4 em agosto. O mesmo não ocorreu no período estiagem, onde seis amostras obtiveram valores inferiores a 0,5 (E1, E2 e E3 em Dezembro e E1, E2 e E3 em janeiro (Fig. 2).

Quantitativamente, o menor valor de densidade encontrado foi 414,36 ind. $\mathrm{m}^{-3}$, na estação 1 , no mês de junho (período chuvoso) e o maior foi 57662,71 ind. $\mathrm{m}^{-3}$ na estação 3 , no mês de julho, também no período chuvoso. Este maior valor foi devido ao predomínio de copepoditos de Oithona (40825,32 ind. $\mathrm{m}^{-3}$ ). Essa densidade de copepoditos de Oithona foi responsável por um pico que ocorreu na estação 3 no mês de junho, o que fez com que a média desta estação no período chuvoso aumentasse. Apesar deste fato, pode-se observar um gradiente crescente da densidade de organismos em direção a jusante, tanto na estação de estiagem como na chuvosa (Fig. 3, tab. 2).

A abundância relativa dos taxa no período seco revelou a predominância de náuplios, seguido de Favella ehrenbergii, os quais juntos chegaram a atingir $88,68 \%$ na estação 2 (Fig. 4). No período chuvoso, a predominância foi de Oithona (copepodito), seguido de perto dos náuplios, que mesmo com valores abaixo dos observados no período seco, chegaram a representar $41,43 \%$ da comunidade na estação 4. Particularmente, Favella ehrenbergii apresentou um gradiente decrescente de abundância da estação 1 para a 4 no período seco, o qual se inverteu no período chuvoso. Já Parvocalanus crassirostris obteve maior abundância nas estações com maior influência marinha (3 e 4).

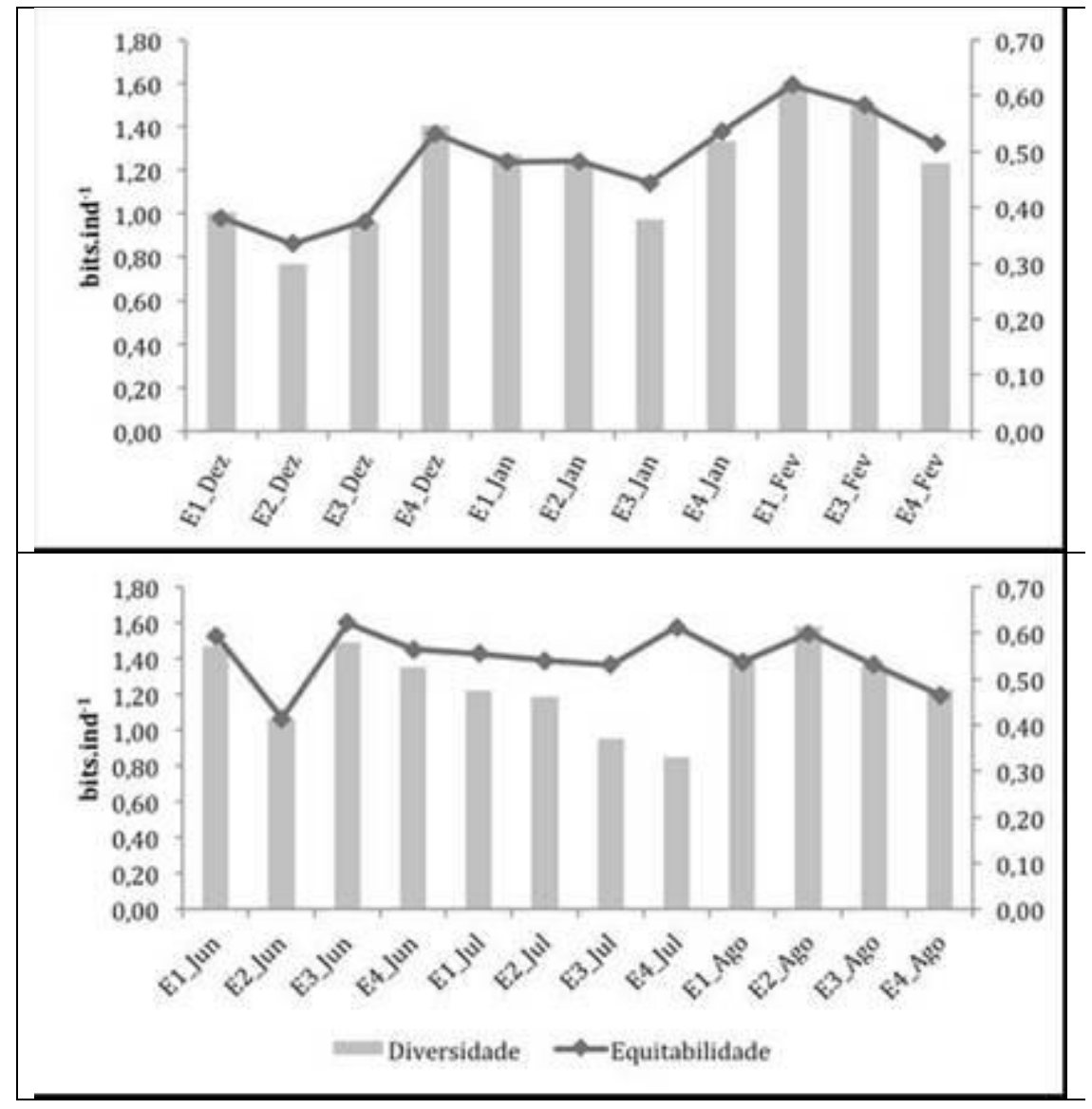

Figura 2 - Diversidade e equitabilidade das amostras coletadas nos períodos de estiagem e chuvoso. 


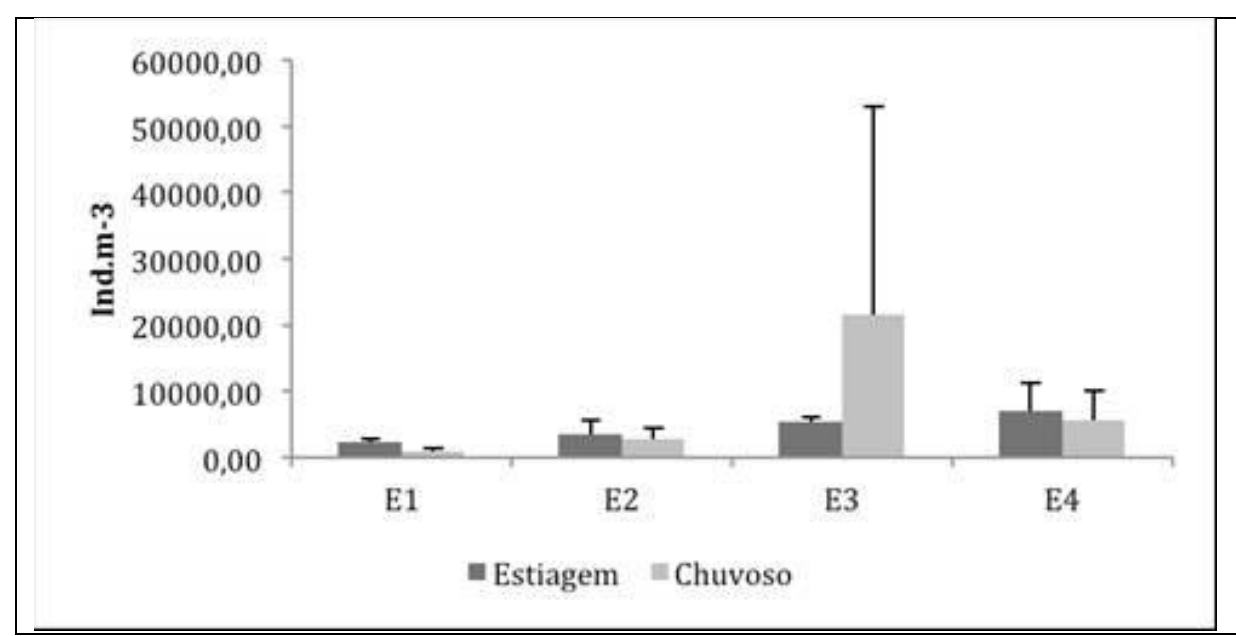

Figura 3 - Média de densidade por estação em cada período (ind. $\mathrm{m}^{-3}$ ).

Tabela 2 - Lista das espécies e de suas densidades (ind. $\mathrm{m}^{-3}$ ) para cada período e em cada estação. Frequência de ocorrência.

\begin{tabular}{|c|c|c|c|c|c|c|c|c|}
\hline \multirow{2}{*}{$\begin{array}{l}\text { PERÍoDo } \\
\text { Táxa Estação }\end{array}$} & \multicolumn{4}{|c|}{ SECO } & \multicolumn{4}{|c|}{ CHUVOSO } \\
\hline & E1 & E2 & E3 & E4 & E1 & $\mathrm{E} 2$ & E3 & $\mathrm{E} 4$ \\
\hline $\begin{array}{l}\text { Foraminifera } \\
\text { (vários) } * * * *\end{array}$ & 10 & 9 & 33 & 72 & 8 & 3 & 13 & 3 \\
\hline Brachionus plicatilis** & & & & & 25 & 92 & 1 & \\
\hline Favella ehrenbergi**** & 589 & 814 & 595 & 570 & 58 & 106 & 279 & 1210 \\
\hline Tintinnina (outros) $* * *$ & 24 & 32 & 12 & 8 & & 2 & 4 & 3 \\
\hline Polychaeta (larva)*** & 2 & 2 & 3 & 5 & 1 & 1 & & 1 \\
\hline Nematoda*** & 11 & & 13 & & 5 & 1 & & 2 \\
\hline Gastropoda (véliger)*** & 4 & 8 & 5 & 4 & 2 & 52 & 20 & 4 \\
\hline Bivalve (véliger)* & & & & 3 & & & & \\
\hline Paracalanus nanus* & & & & & & 2 & & \\
\hline $\begin{array}{l}\text { Parvocalanus } \\
\text { crassirostris**** }\end{array}$ & 11 & 23 & 283 & 556 & 6 & 29 & 202 & 169 \\
\hline $\begin{array}{l}\text { Pseudodiaptomus } \\
\text { (copepodito)** }\end{array}$ & & & 1 & & & & 2 & 3 \\
\hline $\begin{array}{l}\text { Pseudodiaptomus } \\
\text { acutus* }\end{array}$ & & & & 14 & & & & \\
\hline $\begin{array}{l}\text { Pseudodiaptomus } \\
\text { marshi* }\end{array}$ & 1 & & & & & & 1 & \\
\hline $\begin{array}{l}\text { Pseudodiaptomus } \\
\text { richardi* }\end{array}$ & & & 1 & & & & & \\
\hline Acartia lilljeborgi** & & & 4 & 12 & & 2 & & 41 \\
\hline $\begin{array}{l}\text { Oithona } \\
\text { (copepodito)**** }\end{array}$ & 333 & 144 & 457 & 934 & 351 & 1537 & 14258 & 1236 \\
\hline $\begin{array}{l}\text { Oithona } \\
\text { oswaldocruzi**** }\end{array}$ & 151 & 40 & 282 & 541 & 109 & 223 & 2396 & 229 \\
\hline Apocyclops sp.**** & 34 & 66 & 26 & 47 & 4 & 75 & 1093 & 71 \\
\hline Euterpina acutifrons $* * *$ & 6 & & 5 & 4 & 2 & & & 8 \\
\hline Metis holothuriae*** & 47 & 8 & 24 & 1 & 4 & 3 & 5 & 1 \\
\hline Harpacticoida (outros)** & 4 & 2 & 1 & 1 & & & & \\
\hline Monstriloida (vários)* & & & & & & 1 & & \\
\hline Náuplios**** & 1036 & 2310 & 3556 & 4165 & 309 & 691 & 3179 & 2318 \\
\hline Ostracoda*** & 7 & 3 & 3 & & & & & \\
\hline Amphipoda* & & 32 & & & & & & \\
\hline Isopoda*** & 3 & & & 8 & 1 & 18 & & \\
\hline Brachyura (zoea)** & 1 & & 2 & & 1 & 3 & & \\
\hline Oikopleura sp.*** & 4 & 14 & 38 & 28 & & 1 & 12 & 8 \\
\hline
\end{tabular}

$*=$ raro, $* *=$ pouco frequente, $* * *=$ frequente, $* * * *=$ muito frequente 


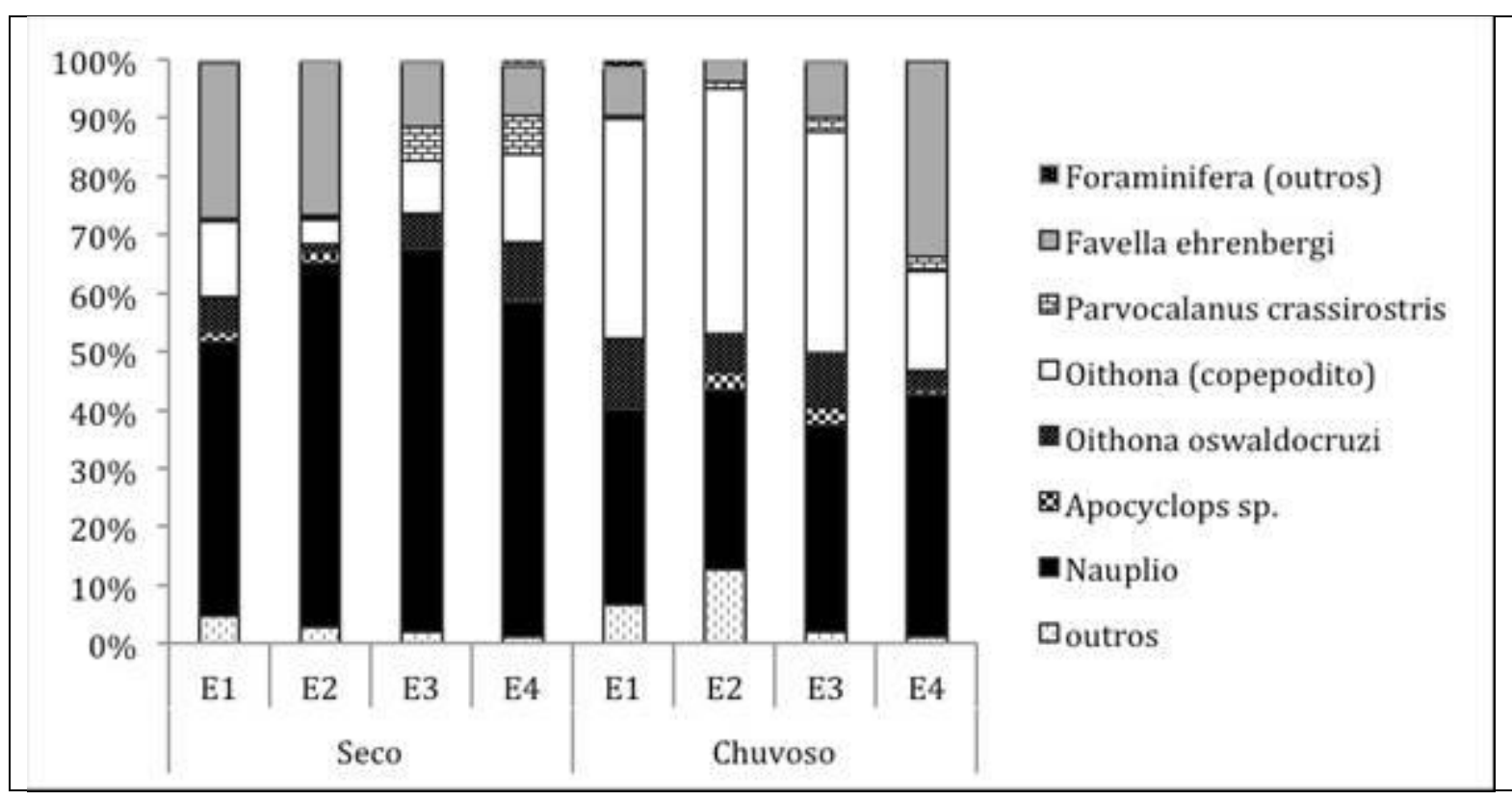

Figura 4 - Abundância relativa (\%) do mesozooplâncton do rio dos Passos, nos períodos de estiagem e chuvoso, em todas as quatro estações de coleta.

\section{DISCUSSÃO}

Nos estuários, de uma maneira geral, as variações sazonais que ocorrem nos fatores ambientais, como salinidade, temperatura, sais nutrientes, turbidez e outros, são influenciadas tanto pela precipitação pluviométrica, como também pelos movimentos periódicos de marés, contribuindo de forma bastante acentuada nas variações hidrológicas características destas regiões (TUNDISI, 1970). Os valores das variáveis hidrológicas encontrados no presente estudo mostraram-se semelhantes aos encontrados por trabalhos realizados tanto no mesmo complexo estuarino do rio dos Passos (HONORATO et al., 2004) como em outros estuários pernambucanos tais como os dos rios Ilhetas e Mamucaba (LOSADA et al., 2000), Barra de Jangadas (BRANCO et al., 2002) e Igarassu (LEÃO et al., 2008).

Os estuários são sempre considerados zonas biológicas ativas (KIBIRIGE; PERISSINOTTO, 2003) devido a intensa produção (primária e secundária) suprida de nutrientes constantemente pelas descargas fluviais (KETCHUM, 1967). Além da disponibilidade de tais nutrientes, variáveis físicas como salinidade e turbidez também influenciam largamente a distribuição e a densidade de comunidades planctônicas em estuários (FERREIRA et al., 2005).

No estuário do rio dos Passos, os teores de clorofila-a estiveram mais elevados durante o período chuvoso, o que pode ser justificado pelo aumento de sais nutrientes derivados da drenagem terrestre pelas chuvas. Neste mesmo sentido, o teor máximo de clorofila-a ocorreu na estação mais a montante no mês de junho, onde foi registrado o maior índice pluviométrico. De acordo com as concentrações de clorofila-a encontrados, o estuário estudado pode ser classificado como oligotrófico (PASSAVANTE, 2003). Desta forma, este fator pareceu não influenciar quantitativamente a comunidade mesozooplanctônica, uma vez que os valores de densidade apresentaram um gradiente de crescimento inversamente proporcional ao da concentração de clorofila-a nas estações amostradas.

O estuário do rio dos Passos sofre grande influência marinha, o que pode ser observado pelo fato de que o valor mínimo de salinidade encontrado foi de 14 , no período e estação com menor influência marinha (período chuvoso, estação mais a montante). Tal estação (E1), foi a de menor média de salinidade, porém, obteve no período de estiagem, uma salinidade máxima de 34. Desta forma, foram predominantes organismos tipicamente estuarinos e costeiros. A entrada desses organismos de altas salinidades ao estuário (e.g. Parvocalanus crassirostris) possivelmente interferiu sobre a densidade, elevando o estoque zooplanctônico das estações 
FIGUEIRÊDO. L. G. P. et AL. Mesozooplâncton do estuário do rio dos Passos, Pernambuco, Brasil.

mais influenciadas pelo oceano gerando um crescimento da densidade em direção a desembocadura, assim como o encontrado por Madhu et al. (2007) em um estuário tropical da Índia. Ainda em consonância com os estudos de Madhu et al. (2007), também foi evidenciado, com a chegada das chuvas, um declínio acentuado da salinidade e portanto da densidade do zooplâncton de origem costeira reduzindo o estoque total.

Se avaliarmos a importância de determinados organismos através de sua contribuição para o fluxo de energia (densidade, biomassa, produção) poderemos evidenciar o valor dos náuplios e das espécies de menor tamanho (e.g. Parvocalanus crassirostris, Oithona oswaldocruzi, Euterpina acutifrons) em seu papel dentro da comunidade zooplanctônica, uma vez que tem sido destacado sua grande produtividade (HOPCROFT et al., 1998) e sua participação essencial (náuplios) como intermediário entre a teia trófica comum e a microbiana (ROFF et al., 1995).

No estuário do rio dos Passos, os náuplios e oithonideos (copepoditos de forma geral e especificamente $O$. oswaldocruzi) estiveram presentes durante todos os meses amostrados, tanto no período de estiagem como no chuvoso, sendo considerados táxons residentes do estuário, evidenciando não somente a chegada destes indivíduos através do fluxo das marés, mas também sua reprodução no local (ESKINAZI-SANT'ANNA, 2000). A ocorrência de $O$. oswaldocruzi no estuário estudado pode ser considerada esperada, por esta ser uma espécie eurihalina tipicamente estuarina e costeira e por estar amplamente distribuída nos ecossistemas costeiros de Pernambuco, tendo ocorrido nos estuários do rio Capibaribe (SILVA et al., 1996), canal de Santa Cruz (PORTO NETO et al., 1999), rio Formoso (NEUMANN-LEITÃO et al., 1995), assim como na plataforma continental (NEUMANN-LEITÃO et al., 1993) entre outros.

Ainda em relação aos Copepoda, o número de espécies encontrado foi pequeno, como também foi encontrado por Neumann-Leitão et al. (1995) no estuário do rio São Francisco. Os Oithonideos dominaram em todas as amostras, chegando a representar $80,75 \%$ da abundancia relativa no mês de julho na estação 3. Dentre os outros taxa de Copepoda encontrados, os Harpacticoida e Metis holothuriae podem ser ditos como meroplanctônicos ou mesmo bênticos, juntamente com outros taxa encontrados, como Polychaeta (larva), Nematoda, Isopoda e Amphipoda, podendo sua ocorrência em camadas mais superficiais da coluna d'água ser atribuída a baixa profundidade local (ESKINAZI-SANT'ANNA, 2000) o que permite a ação de ondas e correntes junto ao fundo, ou mesmo ao deslocamento vertical ativo realizado por alguns desses organismos (CHRISTY; STANCYK, 1982; DAME; ALLEN, 1996).

Entre os Tintinida, Favella ehrenbergii predominou, com ocorrência em todas as estações em todos os meses amostrados. Sua densidade revelou um gradiente crescente conforme aumenta a influência marinha, por ser considerada uma espécie nerítica, restrita a águas rasas (PIERCE; TURNER, 1993) e por altas salinidades favorecerem sua ocorrência (GODHANTARAMAN, 1994). F. ehrenbergii é considerada de grande importância para os estuários de Pernambuco (NEUMANN-LEITÃO et. al., 1996; SILVA, 1997) ocorrendo na bacia do Pina (ESKINAZI-SANT'ANNA; TUNDISI, 1996), e também em outros estados do nordeste brasileiro (ARAUJO, 2008; MAYAL et al., 2009).

Os Rotifera estiveram representados apenas pela espécie Brachionus plicatilis e unicamente no período chuvoso. Esta espécie, diferentemente de outras inclusive do mesmo gênero (i.e. B. rotundiformis) é de grande tamanho (SEGERS, 1995), portanto, uma vez que os Rotifera são coletados mais eficientemente com redes entre 20 a $50 \mu \mathrm{m}$ (NOGRADY et al., 1993) o tamanho de $B$. plicatilis deve ter favorecido sua captura pela rede utilizada neste trabalho $(120 \mu \mathrm{m})$ em detrimento de outras espécies menores.

A ocorrência de $B$. plicatilis apenas no período chuvoso deve-se, mais provavelmente, ser atribuída a queda na temperatura, típica deste período, haja vista que sua produtividade é maior em temperaturas mais reduzidas $\left(<25^{\circ} \mathrm{C}\right)$ (FUKUSHO, 1983), coincidindo sua ocorrência com os meses de menores temperaturas entre as estações de coleta. Ainda, por ser uma espécie eurihalina (FIELDER et al., 2000) e generalista (VANDERPOEG, 1994) a influência de outros fatores como a salinidade e o teor de clorofila-a parecem menos prováveis.

Muitos autores enfatizam a importância dos rotíferos, que podem ser utilizados como 
indicadores de poluição orgânica (RICCI, 1987) por se desenvolverem melhor em áreas eutróficas ou hipereutróficas, onde seu alimento preferido, detritos orgânicos e bactérias, são abundantes (HLAWA; HEERKLOSS, 1994) além de serem atualmente essenciais para a cultura intensiva de larvas de peixes marinhos em viveiros em todo o mundo (KAFUKU; IKENOUE, 1983; LUBZENS, 1987).

A comunidade mesozooplanctônica do estuário do rio dos Passos apresentou de forma geral uma baixa diversidade, o que, de acordo com McLusky e Elliott, (2006) é algo comum para ecossistemas dinâmicos e instáveis a exemplo dos estuários. Resultados semelhantes também foram encontrados em um estudo no sistema estuarino de Barra de Jangada (PESSOA, 2008), onde o autor relacionou tais resultados à poluição industrial e doméstica e por conseguinte redução nas concentrações de oxigênio impedindo o estabelecimento de espécies trazidas pelo fluxo marinho. Fator este, que também pode estar levando a uma distribuição não homogênea do número de indivíduos para os taxa ocorridos no período de estiagem (Equitabilidade $=0,34$ ).

\section{CONCLUSÃO}

O estuário do rio dos Passos apresentou-se sob grande influência marinha, provavelmente devido ao fato deste rio sofrer forte influência do regime de marés. Esta influência afeta a comunidade mesozooplanctônica, definindo a sua composição bem como a distribuição dos organismos ao longo da área estudada. A densidade do mesozooplâncton foi aparentemente mais influenciada pelas variações espaço-temporais da salinidade do que da disponibilidade de alimento (clorofila-a). A diversidade específica apresentada pela comunidade foi baixa, podendo evidenciar um desequilíbrio do ecossistema. Podemos citar porém, que os valores de clorofila-a não indicam processo de eutrofização, o que levanta a possibilidade de outros fatores hidrológicos não estudados neste trabalho estarem relacionados com um possível desequilíbrio desequilíbrio no local de estudo.

\section{AGRADECIMENTOS}

Os autores agradecem ao Laboratório de Fitoplâncton pela disponibilidade de material e equipamento para a realização das analises de clorofila-a, assim como o Laboratório de Zooplâncton pelo suporte nas analises das amostras do mesozooplâncton.

\section{REFERÊNCIAS}

ARAUJO, H. M. P.; NASCIMENTO-VIEIRA, D. A.; NEUMANN-LEITÃO, S.; SCHWAMBORN, R.; LUCAS, A. P. O.; ALVES, J. P. H. Zooplankton community dynamics in relation to the seasonal cycle and nutrient inputs in an urban tropical estuary in Brazil. Braz. J. Biol., v. 68, n. 4, p 751-762, 2008.

AZAM, F.; FENCHEL, T.; FIELD, J. G. et al. The ecological role of water-column microbes in the sea. Mar. Ecol. Prog. Ser. v. 10, p. 257-263, 1983.

BADYLAK, S., PHLIPS, E.J. Spatial and temporal distributions of zooplankton in Tampa Bay, Florida, including observations during a HAB event. J. Plankton Res. v. 30, p. 449-465, 2008.

BJÖRNBERG, T. K. S. Copepoda. in BOLTOVSKOY, D. (Ed). Atlas del zooplâncton del Atlantico Sudocidental y Metodos de trabajo com el zooplancton marino. INIDEP. Mar Del Plata. 1981, $936 \mathrm{p}$.

BOLTOVSKOY, D. Atlas del zooplâncton del Atlantico Sudocidental y Metodos de trabajo com el zooplancton marino. Mar Del Plata: INIDEP. 1981, 936 p.

BOLTOVSKOY, D. South Atlantic zooplankton. Leiden: Backhys. 2v. 1999, 1706 p.

BRANCO, E. S.; FEITOSA, F. A. N.; FLORES MONTES, M. J. Variação sazonal e espacial da biomassa fitoplanctônica relacionada com parâmetros hidrológicos no estuário de Barra das Jangadas (Jaboatão dos Guararapes - Pernambuco - Brasil). Tropical Oceanography. v. 30, p. 79-96, 2002.

CHEN, M., CHEN, B., HARRISON, P.; LIU, H. Dynamics of mesozooplankton assemblages in subtropical coastal waters of Hong Kong: A comparative study between a eutrophic estuarine 
FIGUEIRÊDO. L. G. P. et AL. Mesozooplâncton do estuário do rio dos Passos, Pernambuco, Brasil.

and a mesotrophic coastal site. Continental Shelf Research. v. 31, p. 1075-1086, 2011. CHRISTY, J. H.; STANCYK, S. E. Timing of larval production and flux of invertebrate larvae in a well-mixed estuary. In Kennedy, V.S., ed. Estuarine Comparisons, Academic Press. New York. p. 489-503, 1982.

Companhia Pernambucana do Meio Ambiente (CPRH). Plano de Gestão, Zoneamento Ecológico Econômico Costeiro - ZEEC - APA de Guadalupe Litoral Sul de Pernambuco. Recife, CPRH. 1998.

DAME, R. F.; ALLEN, D. M. Between estuaries and the sea. J. Exp. Mar. Biol. Ecol. v. 200, p. $169-185,1996$.

ESKINAZI-LEÇA, Enide; BARROS-FRANÇA, Lucy Moreira de; MACEDO, Sílvio José de. Estudo ecológico da área de Itamaracá (Pernambuco- Brasil). XXIV. "Standing stock" do fitoplâncoton do estuário do rio Botafogo, durante Janeiro/dezembro/75. Trabalhos Oceanográficos da Universidade Federal de Pernambuco. Recife, v. 18. p. 153-192. 1984.

ESKINAZI-SANT'ANNA, E. M. Zooplankton abundance and biomass in a tropical estuary (Pina estuary-Northeast Brazil). Trabalhos Oceanográficos da Universidade Federal de Pernambuco. Recife, v. 28, n. 1. p. 21-34, 2000.

ESKINAZI-SANT'ANNA, E. M.; TUNDISI, J. G. Zooplâncton do estuário do Pina (RecifePernambuco-Brasil): composição e distribuição temporal. Rev. Bras. Oceanogr., v. 44, n. 1, p. 23-33, 1996.

FERREIRA, J. G.; WOLFF, W. J.; SIMAS, T.C.; BRICKER, S.B. Does biodiversity of estuarine phytoplankton depend on hydrology? Ecological Modeling. v. 187, p. 513-523, 2005.

FIELDER, D.S.; PURSER, G. J.; BATTAGLENE, S. C. Effect of rapid changes in temperature and salinity on availability of the rotifers Brachionus rotundiformis and Brachionus plicatilis.

Aquaculture. v. 189, p. 85-99, 2000.

FLINDT, M. R.; PARDAL, M. A.; LILLEBO, A. I. et al. Nutrient cycling and plant dynamics in estuaries: a brief review. Acta Oecol. v. 20, n. 4, p. 237-248, 1999.

FUKUSHO, K. Present status and problems in culture of the rotifer Brachionus plicatilis for fry production of marine fishes in Japan. In: Fuentes, H. R., Castillo, J. G., Disalvo, L. H. Žeds., Advances and Perspectives in Aquaculture. Proceedings of a Symposium, Universidad del Norte, Coquimbo, Chile, September. p, 361-374, 1983.

GODHANTARAMAN, N. Species composition and abundance of Tintinnids and Copepods in the Pichavaram Mangroves (South India). Ciencias Marinas, v. 20, n. 3, p. 371-391, 1994.

HLAWA, S.; HEERKLOSS, R. Esperimental studies into the feeding biology of rotifer in brackish water. Journal of Plankton Research. v. 16, n. 8, p. 1021-1038. 1994.

HOFFMEYER, M. S. Decadal change in zooplankton seasonal succession in the Bahía Blanca estuary, Argentina, following introduction of two zooplankton species. J. Plankton Res. v. 26, p. 181-189, 2004.

HONORATO-DA-SILVA, M.; PASSAVANTE, J. Z. O.; SILVA-CUNHA, M. G. G.; NASCIMENTOVIEIRA, D. A.; GREGO, C. K. S.; MUNIZ, K. Distribuição espacial e sazonal da biomassa fitoplanctônica e dos parâmetros hidrológicos no estuário do rio Formoso (Rio Formoso, Pernambuco, Brasil). Tropical Oceanography, v. 32, p. 89-106, 2004.

HOPCROFT, R. R.; ROFF, J. C.; LOMBARD, D. Production of tropical copepods in Kingston Harbour, Jamaica: the importance of small species. Marine Biology. v. 130, p. 593-604, 1998.

HUYS, R.; BOXSHALL, G. The orders of copepods. In Huys, R. and Boxshall, G. A. (eds), Copepod Evolution. The Ray Society Series. Ray Society, London, UK. . v. 159, p. 31-314, 1991.

JOHNSON, P. W.; SIEBURTH, J. M. C. N.; XU, H. -S. The utilization of chroococcoid cyanobacteria by marine protozooplankters but not by calanoid copepods. Annales de 
I'Institut Oceanographique. v. 58, p. 297-305, 1979.

KAFUKU, T.; IKENOUE, H. Modern methods of aquaculture in Japan, Developments of Aquaculture and Fisheries Science. Kodansha Tokyo. Elsevier Amsterdam. v. 11, p. 216, 1983.

KENNISH, M. J. Ecology of Estuaries: Biological Aspects. CRC Press. Boca Raton. Florida. v. 2, p. 391, 1990.

KETCHUM, B. H. Phytoplankton nutrients in estuaries. Lau, G.H. (Ed.), Estuaries. American Association for the Advancement of Science. The Horn-Shafer Company. Washington. v. 83, p. 329-335, 1967.

KIBIRIGE, I.; PERISSINOTTO, R. The zooplankton community of the Mpenjati Estuary, a South African temporarily open/closed system. Est. Coast. Shelf Sci. v. 58, p. 727-741, 2003.

LEÃO, M. B.; PASSAVANTE, J. Z. O.; SILVA-CUNHA, M. G. G.; SANTIAGO, M. F. Ecologia do microfitoplâncton do estuário do rio Igarassu, PE, Brasil. Acta Bot. Bras. v. 22, n. 3, 2008.

LOSADA, A. P. M. Biomassa fitoplanctônica relacionada com parâmetros abióticos nos estuários dos Rios Ilhetas e Mamucaba e na Baía de Tamandaré (Pernambuco-Brasil). Recife: 2000. 88f.

Dissertação (Mestrado em Oceanografia) Departamento de Oceanografia. Universidade Federal de Pernambuco.

LUBZENS, E. Raising rotifers for use in aquaculture. Hydro-biologia. v. 147, p. 245-255, 1987.

MADHU, N. V.; JYOTHIBABU, R.; BALACHANDRAN, K. K.; HONEY, U. K.; MARTIN, G. D.; VIJAY, J. G.; SHIYAS, C. A.; GUPTA, G. V. M.; ACHUTHANKUTTY, C. T. Monsoonal impact on planktonic standing stock and abundance in a tropical estuary (Cochin backwaters e India). Estua., Coast. and Shel. Sci. v. 73, p. 54-64, 2007.

MAYAL, E. M.; NEUMANN-LEITÃO, S.; FEITOSA, F. A. N.; SCWAMBORN, R.; SILVA, T. A. \& SILVA-CUNHA; M. G. G. Hydrology, Plankton, and Corals of the Maracajaú Reefs (Northeastern Brazil) - an Ecosystem Under Severe Thermal Stress. Braz. Arch. Biol. Technol. v. 52, n. 3, p. 665-678, 2009.

MCLUSKY, D. S.; ELLIOTT, M. 2006. The estuarine ecosystem, ecology, threats and management. Oxford: Oxford University Press. 214p.

NEUMANN-LEITÃO, Sigrid; GUSMÃO, Lúcia Maria de Oliveira; PARANHOS, Janete Diane Nogueira; NASCIMENTO-VIEIRA, Dilma Aguiar do; PARANAGUÁ, Maryse Nogueira. Zooplâncton da plataforma continental norte do Estado de Pernambuco (Brasil). Trabalhos Oceanográficos da Universidade Federal de Pernambuco, Recife, v. 22, p. 97-116, 1991/93.

NEUMANN-LEITÃO, S.; GUSMÃO, L. M. O.; NASCIMENTO-VIEIRA, D. A.; PARANHOS, J. D. Nogueira. Zooplâncton da Área Estuarina do Rio Formoso-PE (Brasil). Trabalhos

Oceanográficos da Universidade Federal de Pernambuco. Recife, v. 23. p. 55-64, 1994/95.

NEUMANN-LEITÃO, S.; GUSMÃO, L. M. O.; NASCIMENTOVIEIRA, D. A.; NOGUEIRA-PARANHOS, J. D. Variação diurna do zooplâncton no estuário do rio Ipojuca - Pernambuco -Brasil. Trab.

Oceanográficos da Universidade Federal de Pernambuco, Recife, v. 24, p. 103-134, 1996.

NEUMANN LEITÃO, S; SOUZA, M. R. M.; PORTO NETO, F. F.; MOURA, M. C. O.; SILVA, A. P.; GUSMÃO, L. M. O. Zooplâncton do Estuário do Rio São Francisco, Nordeste do Brasil.

Trabalhos Oceanográficos da Universidade Federal de Pernambuco, Recife, v. 27, n. 1, p. 33-54, 1999.

NIVAL, P.; NIVAL, S. Particle retention efficiencies of an herbivorous copepod Acartia clausi (adult and copepodite stages): effects on grazing. Limnology and Oceanography. v. 21, p. 24-38, 1976.

NOGRADY, T.; WALLACE, R. L. \& SNELL, T. W. Rotifera-guides to the identification of the microinvertebrates of the continental waters of the world. SPB Academic Publishing, The Hague. v. 4, p. 142, 1993. 
FIGUEIRÊDO. L. G. P. et AL. Mesozooplâncton do estuário do rio dos Passos, Pernambuco, Brasil.

PASSAVANTE, J. Z. de O. Produção fitoplanctônica do estuário do rio Capibaribe (Recife, Pernambuco, Brasil). In: Congresso Nordestino de Ecologia, 10, 2003, Recife. Anais... 2003. v. CD.

PESSOA, V. T.; MELO, P. A. M. C.; PORTO NETO, F. F.; NEUMANN-LeitÃo, S.; hAZIN, F. H. V. Zooplâncton de Barra de Jangada, Pernambuco, Brasil. Rev. Bras. de Eng. de Pesca. v. 3, n. 3, 2008.

PIERCE, R. W.; TURNER, J. T. Ecology of planktonic ciliates in marine food webs. Rev. Aquat. Sci. v. 6, p. 139-181, 1992.

PIERCE, R. W.; TURNER, J. T. Global biogeography of marine tintinnids. Mar. Ecol. Prog. Ser. v. 94, p. 11-26, 1993.

POOLE, H. H.; ATKINS, W. R. G. Photo - eletric measurements of submarine illumination througouth the year. Journal of the Marine Biological Associotion of the United Kingdon. London, n. 16, p. 297-394, 1929.

ROFF, J. C.; TURNER, J. T.; WEBBER, M. K.; HOPCROFT, R. R. Bacterivory by tropical copepod nauplii: extent and possible significance. Aquat microb Ecol, n. 9, p. 165-175, 1995.

PORTER, K. G.; SHERR, E. B.; SHERR, B. F.; PACE, M.; SANDERS, R. W. Protozoa in planktonic food webs. J. Protozool. v. 32, p. 409-415, 1985.

PORTO NETO, F. F.; NEUMANN-LEITÃO, S; GUSMÃO, L. M. O.; NASCIMENTO VIEIRA, D. A. do; SILVA, A. P.; SILVA, T. A.; Variação Sazonal e Nictemeral do Zooplâncton no Canal de Santa Cruz, Itamaracá, PE, Brasil. Trabalhos Oceanográficos da Universidade Federal de Pernambuco. Recife, v. 27, n. 2. p. 43-58, 1999.

RAMFOS, A.; ISARI, S.; SOMARAKIS, S.; GEORGOPOULOS, D.; KOUTSIKOPOULOS, C.; FRAGOPOULU, N. Mesozooplankton community structure in offshore and coastal waters of the Ionian Sea (eastern Mediterranean) during mixed and stratified conditions. Marine Biology, $v$. 150, p. 29-44, 2006.

RICCI, C. Dormancy patterns in rotifers. Hydrobiologica, v. 446/ 447, p. 1-11, 2001.

ROFF, J. C.; TURNER, J. T.; WEBBER, M. K.; HOPCROFT, R. R. Bacterivory by tropical copepod nauplii: extent and possible significance. Aquat Microb Ecol. v. 9, p. 165-175, 1995.

SASSI, R. Phytoplankton and environmental factors in the Paraiba do Norte river estuary, northeastern Brazil: composition, distribution and qualitative remarks. Boletim do Instituto Oceanográfico de SP, São Paulo, v. 39, n. 2, p. 93-115, 1991.

SEGERS, H. Nomenclatural consequences of some recent studies on Brachionus plicatilis (Rotifera, Brachionidae). Hydrobiologica, v. 313/314, p. 121-122, 1995.

SHANNON, C. E. A mathematical theory of communication. The Bell System Technical Journal. v. 27, p. 379-423 e 623-656, 1948.

SILVA, T. A. ; PARANAGUÁ, M. N.; NEUMANN-LEITÃO, S.; NOGUEIRA-PARANHOS, J. D. Zooplâncton do Estuário do Rio Capibaribe, Recife-PE (Brasil). Trabalhos Oceanográficos da Universidade Federal de Pernambuco. Recife, v. 24, p. 79-102, 1996.

SILVA, A. P. Diversidade, produtividade e dinâmica do microzooplâncton na desembocadura sul do canal de Santa Cruz, Itamaracá-Pernambuco (Brasil). monografia (graduação) -

Departamento de Oceanografia. Universidade Federal de Pernambuco. Recife, 57f. 1997.

STRICKLAND, J. D. H.; PARSONS, T. R. A pratical handbook of sea-water analysis. Bull. Fish. Res. Bd. Can. v. 167. p. 1-11, 1972.

TELESH, I. V. Plankton of the Baltic estuarine ecosystem with emphasis on Neva Estuary: a review of present knowledge and research perspectives. Mar. Pollut. Bull. v. 49, p. 206-219, 2004.

TREGOUBOFF, G.; ROSE, M. Manuel de planctologie Mediterraneéne. Paris: Centre Nacion. 
Sci. v. 2, p. 587, 1957.

TUNDISI, J. G. O plâncton estuarino. Contribuições avulsas do Instituto Oceanográfico de São Paulo, série Oceanografia Biológica, São Paulo, Brasil. v. 19, p. 1-22, 1970.

UNESCO. Determination of photosynthethic pigments in sea water. Report of SCOR/UNESCO Working group 17 with meat from 4 to 6 june 1964 (Monographys on Oceanology Methodology), Paris. 1966, 69 p.

VANDERPOEG, H. A. Zooplankton particle Selection and feeding mechanisms. In: The biology of particles in aquatic systems. Ed. London. Lewis Publishers. v. 2, p. 205-234, 1994. 